\title{
La gestión del conocimiento y la sostenibilidad de la acreditación del Hospital Pablo VI Bosa de Bogotá"
}

\section{Knowledge management and accreditation sustainability of the Pablo VI Bosa Hospital Bogotá}

Recibido: 12 de febrero de 2016 Revisado: 24 de maræo de 2016 Aceptado: 2 de mayo de 2016

Lu: Jeannette Cortés Quiroga"

Hospital Pablo VI Bosa

\section{RESUMEN}

En las instituciones de salud en Colombia no se ha explorado la Gestión del Conocimiento (GC) y su articulación con el Sistema Único de Acreditación en Salud (SUA), como facilitadores efectivos en la creación de ventajas competitivas y el desarrollo de innovación. El objetivo de esta investigación es determinar la relación existente entre la GC de una entidad de salud acreditada de Primer Nivel en Bogotá, y la sostenibilidad del SUA, implementando una propuesta que articule la GG y SUA en la organización. El proyecto corresponde a un estudio mixto concurrente, de tipo descriptivo. Se seleccionó como base la teoría de "creación del conocimiento" (Nonaka y Takeuchi, 2000). Se aplicaron técnicas cuantitativas y cualitativas de recolección de datos: revisión documental, encuestas a colaboradores $(\mathrm{n}=310)$ y entrevistas semiestructuradas a directivos $(\mathrm{n}=5)$. Para el análisis de datos cuantitativos se utilizaron medidas de frecuencia y para las entrevistas análisis de contenido, realizando posteriormente triangulación de resultados. Se realiza una propuesta metodológica de articulación del SUA y la GC en la entidad, con posterior validación a través de los equipos de mejora continua. Como conclusiones, se encuentra que el SUA tiene incorporados elementos de la teoría de Nonaka y Takeuchi que facilitan la creación, captura y transferencia del conocimiento en la entidad, y

\footnotetext{
Artículo resultado de investigación. Actualmente el nombre de la entidad es la Unidad de Servicios de Salud (USS) Pablo VI Bosa, Subred Integrada de Servicios de Salud Sur Occidente - E.S.E.

** Médica cirujana. Especialista en Auditoría en Salud. Magíster (c) en Calidad y Gestión Integral. Líder del proyecto de calidad de la unidad Pablo VI Bosa, Subred Integrada de Servicios de Salud Sur Occidente - E.S.E. Correo electrónico: ljcortes01@hotmail.com.
} 
por tanto promueven el aprendizaje de los colaboradores, facilitando su sostenibilidad. La entidad objeto de estudio tiene un grado de avance en la GC de "moderado a avanzado".

Palabras clave: Gestión del Conocimiento, acreditación, modelo SECI, Sistema Único de Acreditación en Salud, calidad de la atención en salud, facilitadores organizacionales.

\section{ABSTRACT}

Knowledge management and accreditation sustainability of the Pablo VI Bosa Hospital Bogotá. Knowledge Management (KM) and its articulation with the implementation of accreditation systems for health institutions has not been sufficiently explored as a tool in Colombia's health sector. The KM process acts as an effective facilitator in the creation of competitive advantages and innovation. The objective of this research is to determine the existing relationship between KM and an accredited community health institution in Bogotá and the sustainability of accreditation systems using $\mathrm{KM}$ in their organizations. The project is a descriptive mixed concurrent case study that has a theoretical framework focused on the "knowledge creation" theory first proposed by Nonaka and Takeuchi (2000). Both quantitative and qualitative data techniques; documentary research; surveys with staff $(\mathrm{n}=310)$ and semi-structured and directed interviews $(n=5)$. Frequency measurements and content analysis are undertaken are used for the analysis of quantitative data, followed by the triangulation off results. A methodological proposal was then developed on how to introduce KM to the accreditation system within the health institution, followed by evaluation with the continuous improvement teams. As conclusions, the study identified that the health accreditation system already incorporated elements of the knowledge creation theory developed by Nonaka and Takeuchi that facilitate the creation, capture and transfer of knowledge across the health institution and subsequently promote learning among staff, facilitating sustainability. The health institution that participated in the study already has a high level of progress made in the KM scale from "moderate" to "advanced".

Keywords: Knowledge Management, accreditation, model SECI, Unique Health Accreditation System, quality health care, organizational facilitators.

\section{INTRODUCCIÓN}

En las entidades del sector salud en Colombia, la calidad de los servicios que se prestan a la población, para dar respuesta a sus necesidades individuales y colectivas y mejorar su estado de salud, ha sido una preocupación importante. Por tal razón, el Ministerio de Salud y Protección Social (2006) ha establecido el Sistema Obligatorio de Garantía de Calidad en Salud, para responder a estas necesidades, instaurando estándares de alta calidad en uno de sus componentes, el Sistema Único de Acreditación (SUA). Dicho componente es de carácter voluntario en cuanto a su certificación y representa una herramienta de mejoramiento continuo, utilizada como recurso estratégico por las entidades de salud para generar efectividad, mejorar el desempeño y aprendizaje organizacional que lleve a la prestación de servicios de salud de alta calidad (Ministerio de Salud y Protección Social, 2005).

La presente investigación se desarrolla en el Hospital Pablo VI Bosa I Nivel E.S.E., entidad pública de I Nivel de atención de Bogotá - Colombia, (actualmente Unidad de Servicios de Salud Pablo VI, Subred Integrada de Servicios de Salud, Sur Occidente 
E.S.E. $)^{1}$; acreditada en salud desde el año 2006, por el Instituto Colombiano de Normas Técnicas y Certificación - Icontec; siendo la quinta entidad de salud acreditada en el país, de las 34 que en la actualidad cuentan con dicho reconocimiento y de un total de 28.028 Instituciones Prestadoras de Servicios de Salud - IPS inscritas en el Registro Especial de Prestadores de Servicios de Salud - REPS a nivel nacional (Ministerio de la Protección Social, 2017). Este reconocimiento ha llevado a la entidad a asumir retos importantes, como el crecimiento acelerado de la organización, a partir de: la ampliación en el número de sedes asistenciales y administrativas para el desarrollo de nuevos proyectos en salud de cobertura distrital en el marco del modelo de atención primaria en salud, ampliación en el capital humano de la entidad a expensas del personal de contrato y volúmenes importantes de información y conocimiento para implementar y mantener altos estándares de calidad en la prestación de los servicios de salud.

Adicionalmente a los desafíos propios del sector, las actuales condiciones laborales y formas de contratación del talento humano en salud, limitan los procesos de fortalecimiento de competencias y capacidades al personal de contrato, promueven el incremento en las tasas de rotación y, por tanto, la fuga de conocimiento. En este ejercicio de sostenibilidad de la acreditación, se identifica la necesidad de capturar, conservar, utilizar y manejar el conocimiento que se produce en la entidad, de forma eficiente, para lograr un aprendizaje perdurable en el tiempo por parte de sus colaboradores.

En este sentido, una de las estrategias que se ha posicionado a nivel internacional en la sociedad, la

1 A partir del acuerdo 641 del 6 de abril del 2016, "por el cual se efectúa la reorganización del Sector Salud de Bogotá, Distrito Capital, se modifica el acuerdo 257 de 2006 y se expiden otras disposiciones" del Consejo de Bogotá. Se creó la Subred Integrada de Salud Sur Occidente a la que se integró como unidad el Hospital Pablo VI Bosa. economía, las empresas y el mundo académico en general, por ser fuente de poder y cambio de las organizaciones, es la GC. Diferentes autores como Davenport y Prusak (1998, p. 1230); Grant (1996, p. 109); Sveiby (citado por UNE, 2008, p. 8); Benavides y Quintana (2003, p. 27); Tarí y García (2013, p. 151) y Bueno (1999, p. 9), entre otros, coinciden en que la GC es una herramienta estratégica valiosa que lleva a las organizaciones a crear ventajas competitivas sostenibles.

Con base en lo anterior, se puede afirmar que los sistemas de gestión de calidad, para este caso el SUA y la GC, son estrategias esenciales que aportan al desarrollo y logro de los objetivos de las organizaciones, y pueden ser incorporados y desarrollados por las entidades de salud, a fin de garantizar la prestación de servicios de alta calidad. Es así como estudios de Linderman, Schroeder, Zaheer, Liedtke y Choo (2004, p. 590); Easton y Jarrell (1998, p. 225); Camisón, Boronat, Villar y Puig (2008, p. 131); Benavides y Quintana (2003), reportan que los sistemas de gestión de calidad integrados a los de GC, son mecanismos de dirección complementarios e integradores que actúan asegurando una mejora en los resultados de las organizaciones. Adicionalmente, afirman que los sistemas de gestión de calidad contribuyen positivamente a mejorar los sistemas de GC, dado que comparten aspectos o prácticas relevantes como la cultura y el liderazgo. Wiengarten, Fynes, Cheng y Chávez (2013, p. 3056), por su parte, afirman que la gestión por procesos, el ciclo PHVA de Deming, la mejora continua, el aprendizaje, la cooperación interna y externa, dan las condiciones correctas para desarrollar programas de GC e innovación en las organizaciones.

Estudios desarrollados en Bogotá, como el de Ramírez (2011) y Babativa y Hernández (2009) en el marco del convenio USTA - Icontec, reafirman que un sistema de gestión de la calidad facilita la estructuración de un sistema de GC, debido a que es en este primero donde 
se establecen los flujos, procesos, responsables de las actividades y los alcances de la información, los cuales pueden ser referentes para la construcción del sistema de GC.

En este escenario, la investigación asume que para lograr una sostenibilidad de más de 10 años en el proceso de acreditación en salud en un entorno difícil y dinámico, se hace necesario contar con un sistema o prácticas de GC en algún grado de desarrollo, que han permitido a la entidad y a sus colaboradores crear, conservar, utilizar el conocimiento y aprendizaje adquiridos, para desarrollar procesos de innovación en la entidad, mejorar la calidad de los servicios ofrecidos, lograr la satisfacción de los usuarios y de manera consecuente, le permite ser reconocida y conservar la certificación como entidad acreditada.

Sin embargo, debido a que en el sector salud no se han documentado experiencias que ayuden a soportar estos planteamientos desde el SUA, surge la necesidad de comprobar si en salud contiene elementos comunes con la GC que favorecen positivamente su implementación; así como identificar si el Hospital Pablo VI Bosa I Nivel E.S.E. cuenta con una $\mathrm{GC}$ y conocer su grado de avance para favorecer la sostenibilidad de la acreditación.

Las teorías existentes sobre GC abordadas en el marco teórico de esta investigación incluyen: la teoría de capital humano, la teoría del capital intelectual, la teoría de aprendizaje organizacional y la teoría de creación del conocimiento; las cuales han sido desarrolladas a través de modelos como: the 10-Step Road Map (Rodríguez, 2006, p. 32), modelo de KPMG (Angulo y Negrón, 2008), modelo de dirección por competencias (Bueno, 1999), Knowledge Management Assessment Tool (KMAT) (citado por Rodríguez, 2006), modelo de aprendizaje organizativo (Senge, 1992), modelo Andersen (Andersen, 1999 citado por Sánchez, 2005) y el modelo de creación del conocimiento (Nonaka y Takeuchi, 2000).
Sin embargo, en esta investigación se seleccionó la teoría de creación del conocimiento, descrita por Nonaka y Takeuchi por ser una teoría genérica, sistémica, aplicable a todo tipo de organización, ampliamente estudiada, que se centra en la creación del conocimiento partiendo del individuo, aspecto aplicable a entidades de servicio como las del sector salud.

Esta teoría concibe el conocimiento como "una creencia verdadera justificada", y la creación de conocimiento organizacional como el proceso de poner a disposición de la institución el conocimiento creado por los individuos, amplificándolo, cristalizándolo y conectándolo con el sistema de conocimiento de la organización (Nonaka, Von Krogh y Voelpel, 2006, p. 1180) y la GC es asumida como la "capacidad de la empresa para crear conocimiento nuevo, diseminarlo en la organización e incorporarlo en productos, servicios y sistemas" (Nonaka, 1994, p. 15).

La teoría de creación del conocimiento plantea un modelo conformado por dos dimensiones: una epistemológica (conocimiento tácito y explicito) y otra ontológica (individuo, grupo y organización), que contempla cinco pasos: compartir conocimiento tácito, crear conceptos, justificar los conceptos, construir un arquetipo y expandir el conocimiento; el proceso tiene como base la formación de equipos auto-organizables y el establecimiento de espacios para realizar secciones de diálogo grupal, donde los individuos comparten su conocimiento tácito (Rodríguez, 2006, p. 32).

Se identifican en el modelo cinco elementos, descritos como el modelo SECI, el BA, los activos del conocimiento, los facilitadores y una estructura organizacional que incluye la organización hipertexto y el estilo de dirección middle-up-down management que promueven la creación y desarrollo de conocimiento en la organización a través de la creatividad, la innovación y el aprendizaje organizacional (Nonaka, 1994, p. 15). Así mismo establece que el proceso de creación del 
conocimiento se da a través de un pensamiento dialéctico y continuo entre dos conceptos: el conocimiento tácito y el explícito (Nonaka y Takeuchi, 1995, p. 84), el cual es amplificado en una espiral de interacción social entre los individuos, los equipos, la organización y entre organizaciones.

El modelo SECI está conformado por 4 ciclos de conversión del conocimiento (Nonaka, et al., 2006): 1) socialización, a partir del conocimiento tácito a tácito; 2) externalización, a partir del conocimiento tácito al explícito; 3) combinación, de conocimiento explícito a explícito; 4) internalización, de conocimiento explícito a tácito. Por su parte, los "BA" son considerados espacios físicos, virtuales o mentales o una combinación de todos ellos, donde se dan estos ciclos de conversión (BA de origen, BA de interacción, BA cibernético, BA de ejercicio); los activos del conocimiento son definidos como los recursos específicos de la empresa que son imprescindibles para crear los valores de la organización (Nonaka, Toyama y Konno, 2000). Otro elemento es la organización hipertexto, la cual es la estructura ideal para la creación de conocimiento, está constituida por tres capas con actividades interconectadas: burocrática o jerárquica, de proyectos de GC y la base del conocimiento (Nonaka, Von Krogh y Voelpel, 2006, p. 1189).

En cuanto al estilo de dirección que plantea la teoría, es el de centro-arriba-abajo (middle-up-down) (Nonaka et al., 2000), estilo integrador donde los mandos medios tienen un rol estratégico, toda vez que asumen el papel de puente y coordinación entre los equipos operativos y la alta dirección, desarrollando el concepto de rango medio, entre la visión de la gerencia superior (lo que debería ser), y el sentido realista de los empleados de la línea operativa (lo que es) (Nonaka et al., 2006). Finalmente, los facilitadores organizacionales, factores que juegan un papel fundamental para articular y amplificar el conocimiento en el ciclo de conversión: intención, autonomía, fluctuación, redundancia y variedad (Nonaka, Umemoto y Senoo, 1996).
Por su parte, el SUA es un modelo de calidad para la excelencia de las instituciones de salud, basado en el modelo de Joint Commission on Healthcare Organizations (JCHO) (Simón, Guix, Nualart, Surroca y Carbonell, 2001, p. 309). Está reglamentado en Colombia mediante la Resolución 1445 de 2006, modificado en su artículo 2 por la Resolución 123 de 2012, posteriormente con la Resolución 2082 de 2014 y el Decreto 903 de 2014 que la define como:

$$
\begin{aligned}
& \text { El conjunto de procesos, procedimientos y herramientas } \\
& \text { de implementación voluntaria y periódica por parte de las } \\
& \text { entidades a las que se refiere el artículo } 2 \text { del presente } \\
& \text { decreto, los cuales están destinados a comprobar el cum- } \\
& \text { plimiento gradual de niveles de calidad superiores a los } \\
& \text { requisitos mínimos obligatorios, para la atención en sa- } \\
& \text { lud, bajo la dirección del Estado y la inspección, vigilan- } \\
& \text { cia y control de la Superintendencia Nacional de Salud } \\
& \text { (Ministerio de Salud y Protección Social, 2014). }
\end{aligned}
$$

Cuenta con 8 grupos de estándares y 7 ejes (Ministerio de Salud y de Protección Social, 2014).

\section{METODOLOGÍA}

Esta investigación corresponde a un estudio de caso descriptivo, mixto, concurrente que utiliza técnicas de investigación documental, cuantitativas y cualitativas. La investigación se desarrolla en 4 fases o momentos:

\section{Revisión del estado del arte de la GC y del SUA, definiendo sus elementos constitutivos}

A partir de las diferentes teorías y modelos de gestión del conocimiento existentes en la actualidad, se seleccionó como base para este proyecto la teoría creación del conocimiento de Nonaka y Takeuchi (2000).

Se definieron 3 dominios a partir de la revisión de la teoría seleccionada, los cuales agruparon los elementos 
constitutivos de la misma, los dominios son: 1) facilitadores organizacionales; 2) proceso de conversión del conocimiento; 3) infraestructura organizacional; estos a su vez se concentraron en 10 variables, como se presenta en la tabla 1:

Tabla 1. Dominios y variables de la teoría de creación del conocimiento.

\begin{tabular}{|l|l|}
\hline \multicolumn{1}{|c|}{ Dominios o dimensiones } & \multicolumn{1}{c|}{ Variables } \\
\hline \multirow{4}{*}{ Facilitadores organizacionales } & Intención \\
\cline { 2 - 3 } & Fluctuación y caos \\
\cline { 2 - 2 } & Redundancia \\
\cline { 2 - 2 } & Autonomía \\
\cline { 2 - 2 } & Requisito de variedad \\
\hline \multirow{4}{*}{$\begin{array}{l}\text { Proceso de conversión del } \\
\text { conocimiento* }\end{array}$} & Socialización, BA de origen y activos de conocimientos experimentales \\
\cline { 2 - 2 } & Externalización, BA dialogante y activos de conocimientos conceptuales \\
\cline { 2 - 2 } & Combinación, BA cibernético y activos de conocimiento sistemático \\
\cline { 2 - 2 } & Internalización, BA ejercitante y activos de conocimiento de la rutina \\
\hline Infraestructura organizaciona*** & Estilo de dirección (centro-arriba-abajo) y organización hipertexto \\
\hline
\end{tabular}

Fuente: elaboración propia. Adaptado de la teoría de creación del conocimiento (Nonaka y Takeuchi, 2000).

*Se articula la fase de conversión del conocimiento (socialización, externalización, combinación e internalización), el BA asociado y los activos del conocimiento que ingresan y salen durante el proceso, debido a que los 3 elementos se presentan sincrónicamente de manera interdependiente en el proceso de conversión del conocimiento (Von Krogh, Nonanka y Rechsteiner, 2012, p. 241).

**En la infraestructura organizacional se incluyó la organización hipertexto y el estilo de administración centro-arriba-abajo, por ser ambas formas parte del diseño organizacional necesario para la ampliación y difusión del conocimiento.

Asimismo, con base en la normatividad e información disponible sobre el SUA (Ministerio de Salud y de Protección Social, 2014) se definieron tres dominios: 1) metodología de autoevaluación, evaluación y mejoramiento; 2) estándares del Sistema Único de Acreditación; 3) ejes del Sistema Único de Acreditación.

\section{Elaboración de una matriz documental a fin de establecer el grado de concordancia de los elementos del SUA con la GC}

Tomando como base los elementos constitutivos de la teoría de creación del conocimiento y del SUA, se realiza una matriz teórica relacional a fin de establecer y describir su relación en términos de convergencia, divergencia, complementariedad. Se asignó un valor nominal de 0,103 a dicha relación; donde 0 significa que no hay relación; $1=$ hay relación parcial; y $3=$ hay relación total. Para esta investigación se tomará como criterio de aceptación de convergencia los porcentajes superiores o iguales a $80 \%$. 


\section{Diagnóstico del grado de avance de la gestión del conocimiento en la entidad}

Para realizar este diagnóstico se utilizaron técnicas cuantitativas (encuesta), cualitativas (entrevista semiestructurada) y de investigación documental.

Encuesta: el instrumento aplicado correspondió a una encuesta tipo Likert compuesta por 47 ítems con 5 opciones de respuesta. El objetivo de la encuesta era conocer el grado de avance de la GC en el hospital y su relación con el SUA, a partir de la percepción de los colaboradores de la entidad; el instrumento incluía los dominios definidos en la matriz teórica y de manera previa se hizo validación con expertos y prueba piloto con el $10 \%$ de la muestra establecida a través del programa Epi Info ( $\mathrm{n}=32$ colaboradores).

El universo del estudio correspondió a 1.542 colaboradores, donde $1.423(94 \%)$ correspondían a colaboradores de contrato (contrato de prestación de servicios) y 119 colaboradores a personal de planta $(6 \%)$, toda vez que no fueron incluidos 467 al aplicar los criterios de exclusión: a) colaboradores que llevan menos de 6 meses en la organización y b) colaboradores que desarrollan actividades en programas externos al hospital. El tamaño de muestra correspondió a 310 colaboradores y la selección de los mismos se realizó de manera aleatoria a través del programa Microsoft Excel.

Para el análisis de los resultados, se utilizan medidas de frecuencia (proporciones), con énfasis en la opción de respuesta "totalmente de acuerdo" definiendo como nivel de aceptabilidad:

- Favorable = valores iguales o superiores a $50 \%$.

- Moderadamente favorable $=$ resultados entre $40 \% \mathrm{y}$ $49 \%$.
- No favorable = frecuencias inferiores a $39.9 \%$.

Entrevista semiestructurada: entrevista a 5 miembros del equipo directivo del hospital definidos como informantes clave, a fin de comprender y profundizar en su perspectiva y opiniones en relación a la GC existente en la organización, su articulación con el SUA y complementar el diagnóstico haciendo énfasis en la variable relacionada con estilos de dirección - organización hipertexto. Las entrevistas fueron analizadas a través del software N-vivo10.

Investigación documental: a partir del anexo A1 establecido por la Guía práctica española para la gestión del conocimiento - UNE 412001 (Asociación Española de Normalización y Certificación, 2008, p. 89), se definieron las herramientas de GC con las que cuenta la organización de estudio, ubicándolas de acuerdo a su grado de desarrollo y similitud con las planteadas por el anexo de la guía.

Posterior a la recolección de información y al análisis de cada una de las técnicas definidas en el diagnóstico se realizó triangulación de métodos.

\section{Diseño y validación de una propuesta metodológica de GC para la entidad objeto de estudio, articulada al SUA}

El diseño de la propuesta incluyó los elementos y prácticas comunes que se evidenciaron en el diagnóstico, y aquellos relacionados durante la revisión documental y la matriz teórica relacional que no se identificaron en la organización, pero que por su relevancia deben ser tenidos en cuenta. La prueba piloto se realizó a través de los equipos de autoevaluación y mejoramiento continuo del hospital consolidados desde hace años a través de la implementación del SUA. 


\section{RESULTADOS Y DISCUSIÓN}

Los resultados de la investigación están consolidados en 3 secciones: 1) la relación de la GC y el SUA desde un alcance teórico; 2) el diagnóstico del grado de avance de la GC institucional; 3) una propuesta y validación de una metodología que integra la GC y el SUA para mejorar el aprendizaje y la prestación de los servicios de salud en la entidad.

\section{Relación de la GC y el SUA en Salud}

Los resultados encontrados en la matriz de relación teórica confirman la existencia de una correlación entre estos 2 modelos, donde 2 de los dominios muestran resultados favorables de aceptabilidad ( $>80 \%$ ), como se evidencia en la tabla 2:
Tabla 2. Consolidado de resultados por dimensiones de la comparación entre la teoría de creación del Conocimiento y el SUA.

\begin{tabular}{|l|c|}
\hline \multicolumn{1}{|c|}{$\begin{array}{c}\text { Dimensión de la teoría de creación del } \\
\text { conocimiento }\end{array}$} & Porcentaje \\
\hline Facilitadores organizacionales & $87 \%$ \\
\hline Proceso de conversión del conocimiento & $90 \%$ \\
\hline Infraestructura organizacional & $4 \%$ \\
\hline
\end{tabular}

Fuente: elaboración propia. Adaptado de la teoría de Nonaka "creación del conocimiento".

En la revisión específica por variables, se encontró que de las 10 evaluadas de la teoría de creación del conocimiento, 7 se encuentran incorporadas en el SUA con un nivel de aceptabilidad superior al $80 \%$. Como lo muestra la tabla 3:

Tabla 3. Consolidado de resultados por variables según matriz relacional entre el SUA y GC.

\begin{tabular}{|l|l|c|}
\hline \multicolumn{1}{|c|}{ Dominios de la GC } & \multicolumn{1}{|c|}{ Variable de GC } & Grado de relación del SUA \\
\hline \multirow{5}{*}{ Facilitadores organizacionales } & Intención & $96 \%$ \\
\cline { 2 - 3 } & Autonomía & $89 \%$ \\
\cline { 2 - 3 } & Redundancia & $96 \%$ \\
\cline { 2 - 3 } & Fluctuación y caos creativo & $70 \%$ \\
\cline { 2 - 3 } & Requisito de variedad & $81 \%$ \\
\hline \multirow{3}{*}{ Proceso de conversión del conocimiento } & Socialización & $85 \%$ \\
\cline { 2 - 3 } & Externalización & $74 \%$ \\
\cline { 2 - 3 } & Combinación & $100 \%$ \\
\cline { 2 - 3 } & Internalización & $100 \%$ \\
\hline Infraestructura organizacional & Estilos de dirección - organización hipertexto & $4 \%$ \\
\hline
\end{tabular}

Fuente: Elaboración propia.

Las variables externalización $(74 \%)$ del proceso de conversión del conocimiento, fluctuación y caos creativo $(70 \%)$ como facilitador organizacional para el conocimiento y la infraestructura organizacional desde el estilo de administración centro-arriba-abajo (4\%) fueron las más bajas evaluadas.
Las variables de GC que presentaron mayor correlación con el SUA son la combinación (100\%), internalización $(100 \%$ ), intención ( $96 \%$ ) y redundancia (96\%). Estas variables están asociadas con la conversión del conocimiento explícito, la articulación de los estándares del SUA con el direccionamiento estratégico, una 
visión compartida en torno al conocimiento, así como con estrategias de comunicación, información y diálogo entre los diferentes grupos de interés.

Estos resultados guardan estrecha relación con los diferentes estándares del SUA que llevan al establecimiento de abundantes flujos y canales de información en la entidad, el enfoque en procesos, el afianzamiento en los valores y principios éticos, la estandarización de las rutinas institucionales, la identificación de necesidades y expectativas de los clientes y grupos de interés, la elaboración de guías de manejo, protocolos, planes de mejora, minería y análisis de datos para la toma de decisiones, en especial lo reflejado en los estándares de gerencia de la información, direccionamiento, mejora continua y atención del cliente asistencial.

Así mismo, se lograron identificar otras prácticas que apoyan esta convergencia desde el SUA, como son: el trabajo en equipo y la conformación de equipos de trabajo, la filosofía y metodología de mejoramiento continuo a través del ciclo PHVA, los ejercicios de referenciación comparativa y competitiva (interna 0 externa), lecturas periódicas del entorno, el fomento de la relación docente-asistencial, la investigación en las entidades acorde a su nivel de complejidad, el fomento de la transformación de la cultura organizacional, la orientación a la innovación y la realización periódica de autoevaluación del SUA; esta última que sirve como vehículo para la creación de conocimiento y aprendizaje institucional (Balbastre, 2001, p. 224), aportando a un activo del conocimiento como es la memoria organizacional.

\section{Diagnóstico organizacional de GC}

A través del diagnóstico se pudo determinar que el Hospital Pablo VI Bosa E.S.E, tiene elementos y herramientas importantes que lo ubican en un nivel de desarrollo medio-avanzado en cuanto a la GC. Afirmación que tiene su sustento en los resultados obtenidos tanto en las encuestas, entrevistas y revisión teórica sobre las herramientas para la GC, donde se evidencia que se encuentra implementado en diferentes grados de avance: los facilitadores organizacionales, los ciclos de conversión, los estilos de liderazgo, así como las herramientas o activos del conocimiento. Todos estos, elementos constitutivos de la GC descritos en la teoría de Nonaka y Takeuchi (2000).

\section{Percepción de los colaboradores (encuesta)}

El nivel de avance de la organización en la GC se soporta por los resultados encontrados en la encuesta, donde 35 de las 47 afirmaciones se hallan en niveles de aceptabilidad de moderado (40\% a $49 \%$ ), un $36 \%$ de las afirmaciones a superior (> o igual a $50 \%$ ), $38 \%$ de las afirmaciones en la opción de respuesta totalmente de acuerdo. 
Tabla 4. Consolidado de resultados por dominios y variables de GC, según encuestas.

\begin{tabular}{|l|l|c|c|}
\hline \multirow{5}{*}{ Dominios de GC } & \multicolumn{1}{|c|}{ Variables GC } & N. ${ }^{\circ}$ colaboradores totalmente de acuerdo & $\%$ \\
\hline \multirow{5}{*}{ Proceso de conversión del conocimiento } & Socialización & 152 & $49 \%$ \\
\cline { 2 - 5 } & Externalización & 131 & $42 \%$ \\
\cline { 2 - 5 } & Combinación & 161 & $52 \%$ \\
\cline { 2 - 5 } & Internalización & 170 & $55 \%$ \\
\hline \multirow{5}{*}{ Facilitadores organizacionales } & Intención & 180 & $58 \%$ \\
\cline { 2 - 5 } & Autonomía & 97 & $31 \%$ \\
\cline { 2 - 5 } & Redundancia & 125 & $40 \%$ \\
\cline { 2 - 5 } & Fluctuación y caos creativo & 186 & $60 \%$ \\
\cline { 2 - 5 } & Requisito de variedad & 129 & $42 \%$ \\
\hline Infraestructura organizacional & Estilos de administración & 141 & $45 \%$ \\
\hline
\end{tabular}

Fuente: elaboración propia.

Las variables con mayor afianzamiento en el dominio "proceso de conversión del conocimiento" son la combinación e internalización; en cuanto al dominio "facilitadores organizacionales", sobresale la fluctuación y caos creativo y la intención.

En relación a los resultados por afirmación, sobresale la percepción de sentirse identificados y compartir plenamente la visión, misión y objetivos del hospital (78.1 $\%$ totalmente de acuerdo), lo que habla de una visión compartida para la GC. En contraste, la afirmación más baja percibida corresponde a la autonomía de los individuos en solucionar imprevistos dentro de su ámbito de trabajo (22.3\% totalmente de acuerdo). Siendo ambos facilitadores organizacionales que unidos a la fluctuación y caos creativo, logran el compromiso de los miembros de la organización.

\section{Percepción de los directivos en la operativización de la GC}

Las entrevistas permitieron ratificar desde el sentir de los directivos, los resultados obtenidos en la encuesta.
Percibieron que la estructuración organizacional frente a la GC evidencia el predominio de una organización burocrática, donde a pesar de la existencia de un área dedicada a la GC, esta se encuentra focalizada en temas específicos como la investigación y la sistematización de experiencias, desde la perspectiva de crear memoria institucional. Asimismo, emergió de manera importante en los 5 directivos el compromiso de la alta dirección con la GC expresado como: "se impulsa el tema de la gestión del conocimiento, tanto es así que nuestra última plataforma desde nuestra misión y visión, esta puesta no implícitamente como esa palabra, pero incluye temas como la innovación y la investigación" (E2).

En el caso de las variables de la encuesta evaluadas con porcentajes más bajos, los directivos reconocen como oportunidad de mejora la necesidad de establecer en forma explícita la rotación del personal como fuente de conocimiento, precisar el reconocimiento y estímulos por el aporte a la creación del conocimiento, así como definir y estimular la autonomía individual y grupal en la organización. 
En cuanto al papel de los mandos medios, los informantes clave afirman que tienen la función de fomentar la creación del conocimiento y ser armonizadores de los procesos organizacionales ayudando a integrar la información, políticas, lineamientos emitidos por la alta dirección con lo que piensan, saben y sienten ellos en los servicios.

De igual manera, emerge que el proceso de acreditación tiene gran influencia en la organización por la cultura de mejoramiento continuo de la calidad, que promueve y que ha llevado al aprendizaje institucional, a través de la construcción de procesos y herramientas de GC; donde afirman que existe una cultura fortalecida de "aprender y reaprender", relacionada con el proceso de gestión de la calidad institucional; lo que a su vez ha llevado a la organización a generar novedosos procesos y procedimientos para generar impacto social.

Por último, se puede concluir que la acreditación ha servido a la entidad como herramienta para fomentar, apalancar y construir conocimiento como lo refieren los entrevistados, favoreciendo con ello su sostenibilidad.

\section{Madurez de las herramientas de GC con que cuenta la organización}

A través de la matriz documental se analizaron 66 herramientas o prácticas de GC, las cuales 60 son propuestas por la Guía práctica UNE (Asociación Española de Normalización y Certificación, 2008, p. 84) y 6 se identificaron por parte de la investigadora como adicionales en la organización de estudio.

Los resultados del análisis de las herramientas para la GC clasificadas según el grado de madurez muestra que la organización tiene un nivel de madurez baja en un $42 \%$; medio en un $35 \%$ y avanzado en un 23 $\%$, resultados que apoyan la categorización del grado de desarrollo de la entidad, donde se destacan herramientas institucionales de nivel avanzado como: los sistemas e-learning para formación del personal, espacios de comunicación e información: Unidades Locales de Comunicación (ULC), Unidades de Análisis Temático (UAT), Unidades Locales Gerenciales (ULG) y Pablipioneros. Otras de las herramientas de nivel avanzado con que cuenta la organización son el sistema extranet (página web), wikis, software de análisis cuantitativo y cualitativo y plan de gerencia de la información, principalmente.

Dentro de las herramientas de GC de madurez organizativa media con las que cuenta la entidad se resaltan: redes de colaboración y conocimiento, los equipos de mejoramiento continuo, comités ad-hoc, equipos de briefing y debriefing, repositorio de referenciación comparativa institucional, aplicativos in house e historia clínica sistematizada.

\section{Propuesta metodológica para articular la GC y SUA}

A partir del diagnóstico realizado, se estableció una propuesta de GC articulada al SUA; su implementación y evaluación en la entidad evidenció que al introducir la estructura organizacional de tipo hipertexto se armoniza y facilita la GC, a través del empoderamiento de los equipos de autoevaluación y mejora continua del SUA ya existentes, transformándolos en equipos de innovación y gestión del conocimiento.

Asimismo, se estableció un equipo líder de GC integrando a todas las áreas que participan directamente en el proceso en la entidad; esto facilitó la articulación, estructuración, participación y concientización de los colaboradores en torno a la GC; de igual manera se concretó una política más clara en relación a la GC en la entidad para el logro de los objetivos. La validación de la propuesta se realizó a través de los equipos de mejoramiento continuo, promoviendo espacios de interacción social en aspectos relacionados con el 
diálogo y comunicación cara a cara entre los integrantes para fortalecer el compartir de conocimiento tácito y explícito.

Los resultados obtenidos en la presente investigación aportan en el abordaje del vacío del conocimiento existente en el sector salud, siendo esta investigación una primera aproximación a este análisis en relación a la implementación de la GC y el SUA de forma articulada, como estrategias eficientes, que brindan a las organizaciones posibilidades de innovación y ventajas competitivas, favoreciendo el desempeño organizacional y la prestación de servicios de salud de calidad.

Lo anterior confirma los planteamientos realizados en otras investigaciones por Linderman et al. (2004); en lo concerniente a que estos 2 sistemas son mecanismos de dirección complementarios e integradores que actúan asegurando una mejora en los resultados de las organizaciones (Linderman et al., 2004; Camisón, Boronat, Villar y Puig, 2008; Benavides y Quintana, 2003).

Por otra parte, los resultados encontrados en la matriz teórica (tabla 2) y en el diagnóstico de la organización (tabla 3) son consistentes, en primera instancia en las variables del proceso de conversión del conocimiento, donde las 4 variables presentaron comportamientos similares, en relación a su escala de valoración, predominando la combinación e internalización. Como lo plantea Nonaka et al. (2006), la combinación está relacionada con el procesamiento y manejo de la información (conocimiento explícito), aspecto característico en la metodología y estándares del SUA; mientras que la internalización está asociada con el aprendizaje organizacional, propósito central del SUA.

En el dominio "facilitadores organizacionales", la variable intención fue predominante tanto en el diagnóstico de la organización como en la matriz teórica. Por su parte, las variables fluctuación y caos, autonomía, redundancia y estilos de dirección, presentaron diferencias en los resultados obtenidos en la matriz teórica frente al diagnóstico institucional. Este comportamiento puede estar relacionado con aspectos externos al SUA como la cultura organizacional. En ese sentido, en el diagnóstico de cultura institucional realizado en 2013 por la entidad objeto de estudio, se identificó que es una institución con alta orientación al logro, estándares de vocación de servicio y calidad en el core del negocio (Assesment and Business, 2013), razones que pueden justificar la alta aceptabilidad en la variable fluctuación y caos en el diagnóstico organizacional de GC.

Por otro lado, los equipos de autoevaluación y mejora que plantea la metodología del SUA favorecen la creación y GC como plantea Nonaka (1994, p. 32) en relación al rol que desempeñan estos equipos de trabajo en el proceso de creación de conocimiento; al compartir espacios físicos y virtuales (BA) donde se genera la conversión del conocimiento en sus 4 ciclos, dando interacción entre el conocimiento tácito y explícito entre sus integrantes que será desplegado posteriormente en los diferentes procesos de la organización. Aspecto evaluado positivamente tanto en la matriz teórica como en el diagnóstico institucional.

Adicionalmente, al relacionar las afirmaciones de Balastre (2001); Calvo, Navarro y Periañez (2015); Camisón et al. (2009); Durán, Cetinder y Sahan (2014), en cuanto a que el modelo EFQM de excelencia y los modelos de gestión de calidad total (en los que se puede incluir al SUA), pueden ser un marco válido en los que se puede implementar un proceso de GC, porque hacen explicito el aprendizaje y la GC para la mejora continua de la organización, aspecto que es evidente en el SUA; permitiendo vincular el proceso de la autoevaluación con el aprendizaje y la creación de conocimiento organizacional.

Finalmente, en el dominio "estructura organizacional", las variables organización hipertexto y estilos de dirección no se presentan de manera explícita en 
el SUA; sin embargo, en la organización se evidenció que predomina una organización burocrática con fuerte visión del rol de los líderes tácticos, lo cual favorece positivamente la creación de conocimiento como lo describe Nonaka y Takeuchi (2000).

\section{CONCLUSIONES}

A partir de los resultados de la matriz teórica, se concluye que existe una correlación entre los elementos de la Teoría de Creación del Conocimiento y el Sistema Único de Acreditación, lo que a su vez permite inferir que el SUA tiene incorporadas prácticas y estrategias de gestión del conocimiento que facilitan su implementación; comprobándose afirmativamente una de las hipótesis planteadas en este estudio.

A través del diagnóstico se determinó que el Hospital Pablo VI Bosa E.S.E, tiene elementos y herramientas importantes que lo ubican en un nivel de desarrollo medio-avanzado en cuanto a la GC, dentro de las que se resaltan: los equipos de mejora continua, los espacios de comunicación (ULC, ULG, UAT, briefing y debriefing), así como las TIC (aplicativos in house, historia clínica sistematizada, software, intranet, elearning, redes sociales), ejercicios de referenciación comparativa y competitiva, lectura sistemática del entorno mejora continua a través del ciclo PHVA y repositorios, principalmente.

En el diagnóstico realizado en la entidad seleccionada, se evidencia que predomina la transformación del conocimiento explícito, organización burocrática características de los sistemas de GC en la cultura occidental (Nonaka, Unemoto, y Senoo, 1996, p. 216).

El SUA a través de sus estándares, ejes y metodología, promueve el desarrollo de prácticas de GC relacionadas con los facilitadores organizacionales sugeridos por Nonaka y Takeuchi, los cuales juegan un papel fundamental en la integración y amplificación del conocimiento en el ciclo de conversión, permitiendo el desarrollo de la espiral de la creación del conocimiento en la organización.

Finalmente, la encuesta utilizada en el diagnóstico realizado, es un instrumento que puede ser aplicado en otras organizaciones del sector salud que implementen el SUA dado el proceso de validación desarrollado durante la investigación.

Se recomienda al Ministerio de Salud y Protección Social y al ente acreditador en Colombia, Icontec, la incorporación de estándares específicos de GC en el SUA, toda vez que cuenta con muchos elementos incorporados en su metodología que facilitan el proceso. Uno de los estándares a incluir en el SUA estaría relacionado con estructurar desde el direccionamiento estratégico, la GC a través de una política institucional que permita su identificación, gestión, transferencia y evaluación periódica a fin de mejorar la prestación de servicios de salud de calidad. Otro de los estándares que se sugiere incluir en el SUA a partir de los resultados de esta investigación, es la implementación de los facilitadores y la estructura organizacional requerida para gestionar el conocimiento, de modo que facilite la creación e innovación de prácticas, procedimientos y acciones que mejoren el estado de salud de la población a nivel nacional.

Asimismo, en forma específica, se sugiere complementar los estándares del SUA con elementos y prácticas de GG que hagan más explícita su importancia, como: incrementar la captura y fomento del conocimiento tácito de los colaboradores, producir en forma intencional la sensación de caos o averías periódicas para romper con la rutina, promover la rotación del talento humano en forma estructurada como estrategia que aporta a la creación de competencias en los empleados de la organización. Del mismo modo, fomentar el cambio a una 
estructura organizacional hipertexto y la promoción de un estilo de dirección "centro-arriba-abajo", donde los mandos medios tienen un rol estratégico, toda vez que asumen el papel de puente y coordinación de los equipos entre la visión de la gerencia (lo que debería ser) y el sentido realista de los empleados de la línea operativa (lo que es), ayudando de esta forma a la canalización, articulación y amplificación del conocimiento y a la promoción de una alta atmósfera de cuidado, motivación y confianza entre el equipo.

A nivel institucional, se recomienda fortalecer la estructura organizacional de la GC, articulando áreas y procesos de la organización que aportan al tema, a partir del área ya constituida por la entidad para tal fin; así como crear conciencia en todos los colaboradores y conceptualizar de forma explícita sobre la orientación que tiene el hospital en relación a la GC de la entidad y la importancia de su participación en el desarrollo del proceso a partir de las rutinas organizacionales.

Igualmente, diseñar, implementar y evaluar estrategias que promuevan una cultura organizacional colaborativa orientada al conocimiento, que permita fortalecer la confianza y el compartir entres sus miembros; concretar la intencionalidad de la GC en la organización a través de una política clara articulada al código de ética del hospital.

Organizar la estructura para la GC en los equipos de mejoramiento organizacional del SUA, ejerciendo un liderazgo situacional, minimizando en esos espacios la estructura burocrática típica de las organizaciones.

De la misma manera, fortalecer las estrategias que exploren, compartan y utilicen más el conocimiento tácito de los colaboradores, visto desde las habilidades, destrezas y conocimiento adquirido a través de la experiencia por cada uno de ellos; y diseñar e implementar estrategias que favorezcan la autonomía de los colaboradores y los grupos de trabajo en la organización.

\section{Líneas futuras de investigación}

a. Evaluar a mediano plazo, a través de procesos de investigación, el grado de afianzamiento y resultados en la implementación de la metodología de GC propuesta en la entidad de estudio articulada al SUA.

b. Realizar estudios con otras entidades acreditadas a nivel nacional, para evaluar la relación existente entre el grado de desarrollo de la GG y la sostenibilidad del SUA.

c. Implementar la propuesta metodológica presentada en entidades no acreditadas, a fin de facilitar su certificación en el SUA.

d. Evaluar en estudios posteriores el mantenimiento del nivel alcanzado por la entidad dentro del modelo de salud actual vigente por subredes a nivel distrital, y qué tanto del conocimiento se pudo perder por la forma de contratación de los colaboradores y la unión de diferentes entidades con niveles disímiles de avance en el SUA.

e. Explorar en una próxima etapa de investigación, los aportes en la gestión del conocimiento de estas organizaciones de otros modelos de producción del conocimiento en investigación, como el modo 2 (Gibbons et al., 1997) y el modo 3 de creación y construcción de conocimiento (Acosta y Carreño 2013) utilizado en espacios universitarios.

\section{REFERENCIAS}

Acosta V., y Carreño, M. (2013). Modo 3 de producción de conocimiento: implicaciones para la universidad de hoy. Revista Universidad de la Salle. Bogotá. Recuperado de https://revistas.lasalle.edu.co/index.php/ls/article/view/2439 
Angulo, E., y Negrón, M. (2008). Modelo holístico para la gestión del conocimiento. Revista Científica Electrónica Ciencias Gerenciales, 11(4), 38-51. Recuperado de https://www.revistanegotium.org.ve/ pdf/11/Art2.pdf

Asociación Española de Normalización y Certificación. (2008). Guía práctica de la Gestión del Conocimiento. Madrid: AENOR.

Assesment and Business. (2013). Diagnóstico de Cultura Organizacional - Hospital Pablo VI Bosa I Nivel E.S.E. Bogotá, Colombia.

Babativa, O., y Hernández, M. (2009). Determinación y evaluación de la gestión del conocimiento en una organización del sector farmacéutico nacional. Tesis de maestría en Calidad y Gestión Integral. Bogotá: Universidad Santo Tomás.

Balbastre, F. (2001). La autoevaluación según los modelos de gestión de calidad total y el aprendizaje en la organización: una investigación de carácter exploratorio. Tesis doctoral. Universidad de Valencia.

Balbastre, F. (julio de 2001). La autoevaluación segín los modelos de gestión de calidad total y el aprendizaje en la organización: una investigación de carácter exploratorio. Tesis doctoral. Valencia: Universidad de Valencia.

Benavides, C., y Quintana, C. (2003). Gestión del Conocimiento y calidad total. Madrid: Ediciones Díaz de Santos.

Bueno, E. (1999). La Gestión del Conocimiento: Nuevos perfiles profesionales. Boletín Del Club Intelectual, 18. Recuperado de http://www.academia. edu/8013246/LA_GESTI\%C3\%93N_DEL_CONOCIMIENTO_NUEVOS_PERFILES_PROFESIONALES
Calvo, M., Navarro, G., \& Periañez, C. (2015). Project to improve knowledge management and key business results through the EFQM excellence model. International Journal of Project Management, 33(8), 1638-1651. Recuperado de https://doi. org/10.1016/j.ijproman.2015.01.010

Camisón, C., Boronat, M., Villar, A., y Puig, A. (10 de marzo de 2008). Sistemas de gestión de la calidad y desempeño: importancia de las prácticas de gestión del conocimiento y de I+D. Revista Europea de Dirección y Economía de la Empresa, 18(1), 123134. Recuperado de https://dialnet.unirioja.es/ descarga/articulo/2878071.pdf

Davenport, T. H., \& Prusak, L. (1998). Working Knowledge: How Organizations Manage What They Know. Knowledge Creation Diffusion Utilisation, 309. Recuperado de https://doi.org/10.1109/ EMR.2003.1267012

Durán, C., Çetindere, A., \& Şahan, Ö. (2014). An analysis on the relationship between total quality management practices and knowledge management: The case of Eskişehir. Procedia-Social and Behavioral Sciences, 109, (pp. 65-77). Recuperado de https://doi.org/10.1016/j.sbspro.2013.12.422

Easton, G., \& Jarrell, S. (2014). Investigation The Effects of Total Quality Management on Corporate Performance : An Empirical Investigation *, 71(2), 253-307. Recuperado de: http://www.jstor. org/stable/10.1086/209744

Gibbons, M., Limoges, C., Nowotny, H., Schwartzman, S., Scott, P., y Trow, M. (1997). La nueva producción del conocimiento. La dinámica de la ciencia y la investigación en las sociedades contemporáneas. Barcelona: Ediciones Pomares-Corredor S. A. Recuperado de http://www.ses.unam.mx/ docencia/2006II/gibbonslec.pdf 
Grant, R. (1996). Toward a knoweldge-based theory of the firm. Strategic Management Journal, 17(S2), 109-122. Recuperado de https://doi.org/10.1002/ smj.4250171110.

Linderman, K., Schroeder, R., Zaheer, S., Liedtke, C., \& Choo, A. (2004). Integrating quality management practices with knowledge creation processes. Journal of Operations Management, 22(6).

Ministerio de la Protección Social. (11 de abril de 2017). Registro Especial de Prestadores de Servicios de Salud - REPS. Recuperado de https://prestadores. minsalud.gov.co/habilitacion/

Ministerio de Salud y de Protección Social. (13 de mayo de 2014). Decreto 903: por el cual se dictan disposiciones en relación con el Sistema Único de Acreditación en Salud. Recuperado de https://www. minsalud.gov.co/sites/rid/Lists/BibliotecaDigital/ RIDE/DE/DIJ/Decreto-0903-de-2014.pdf

Ministerio de Salud y de Protección Social. (29 de mayo de 2014). Resolución 2082:por la cual se dictan disposiciones para la operatividad del Sistema Único de Acreditación en Colombia. Recuperado de http:// www.acreditacionensalud.org.co/sua/Documents/ Resoluci\%C3\%B3n\%202082\%20de\%202014.pdf

Ministerio de Salud y Protección Social. (2005). Guía práctica de preparación para la acreditación en salud. Bogotá: Impresor.

Ministerio de Salud y Protección Social. (26 de enero de 2012). Resolución 123: por la cual se modifica el articulo 2 de la Resolución 1445 de 2006. Recuperado de http://www.acreditacionensalud.org.co/sua/ Documents/Res123\%20ene26de2012\%20modificRes1445.pdf

Ministerio de Salud y Protección Social. (3 de abril de 2006). Decreto 1011: por el cual se establece el Sistema
Obligatorio de Garantía de la Calidad de la Atención de Salud del Sistema General de Seguridad Social en Salud. DO: 46.230. Bogotá.

Nonaka, I. (1994). A dynamic theory of organizational knowledge creation. Organization science, 5(1), 15.

Nonaka, I., Umemoto, K., \& Senoo, D. (1996). From information processing to knowledge creation: A paradigm shift in business management. Technology in Society, 18(2 SPEC. ISS.), 203-218. Recuperado de https://doi.org/10.1016/0160-791X(96)00001-2

Nonaka, I., Unemoto, K., \& Senoo, D. (1996). From information processing to knowledge creation: a paradigm shift in business management. Technology in society, 18(2).

Nonaka, I., Von Krogh, G., \& Voelpel, S. (2006). Organisational Knowledge Creation Theory: Evolutionary Paths and Future Advances. Organization Studies (Vol. 27). Recuperado de https://doi. org/10.1177/0170840606066312

Nonaka, y Takeuchi. (2000). La empresa creadora de conocimiento. Gestión del conocimiento. Harvard Business Revisado en: Gestión del conocimiento. Germán Orbegozo (Trad.). Bilbao. Ediciones Deusto, 2000, vol. 200, p. 23-49.

Nonaka; Toyama; y Konno. (2000). SECI, Ba and leadership: a unified model of dynamic knowledge creation. Long range planning, vol. 33, no 1, p. 5-34.

Ramírez, M. (2011). La Gestión del Conocimiento y su relación con la Gestión de la Calidad y la Excelencia. Tesis de maestría Calidad y Gestión Integral. Bogotá: Universidad Santo Tomás.

Rodríguez, G. (2006). Modelos para la creación y gestión del conocimiento: una aproximación teórica. Bogotá: Editorial Educar. 
Sánchez, D. (2005). Breve inventario de los modelos de gestión del conocimiento de las organizaciones. Recuperado de http://scielo.sld.cu/scielo.php?script=sci_art text\&pid=S1024-94352005000600006

Senge, P. (1992). La Quinta Disciplina. El arte y la práctica de la organisación abierta al aprendiঞaje. Buenos Aires: Ed. Granica Vergara.

Simón, R., Guix, J., Nualart, L., Surroca, R., y Carbonell, J.-M. (2001). Utilización de modelos como herramienta de diagnóstico y mejora de la calidad: EFQM y Joint Commission. Revista de Calidad Asistencia, 16(5), 309.

Tarí, J., y García-F. (2013). ¿Puede la gestión del conocimiento influir en los resultados empresariales?
Cuadernos de Gestión, 13(1), 151-176. https://doi. org/10.5295/cdg.100263jt

Von Krogh, G., Nonanka, I., \& Rechsteiner, L. (2012). Leadership in organizational knowledge creation: A review and framework. Journal of Management, 49(1).

Wiengarten, F., Fynes, B., Cheng, E., \& Chavez, R. (2013). Taking an innovative approach to quality practices: exploring the importance of a company's innovativeness on the success of TQM practices. International Journal of Production research, 51(10), 3055-3074. 